\title{
High-hyperopia database, part l: clinical characterisation including morphometric (biometric) differentiation of posterior micro- phthalmos from nanophthalmos
}

${ }^{1}$ Srimati Kannuri Santhamma Centre for Vitreo- Retinal Diseases, Kallam Anji Reddy Campus, L V Prasad Eye Institute, Hyderabad, India

${ }^{2}$ Kode Venkatadri Chowdary, David Brown Children's Eye Care Centre, L V Prasad Eye Institute, Tadigadapa, Vijayawada, India

${ }^{3}$ Center for Clinical Epidemiology and Biostatistics, Kallam Anji Reddy Campus, L V Prasad Eye Institute, Hyderabad, India

Correspondence: S Jalali, Srimati Kannuri Santhamma Centre for Vitreo- Retinal Diseases, Kallam Anji Reddy Campus, L V Prasad Eye Institute, Banjara Hills, L V Prasad Marg, Hyderabad 500034, India

Tel: +91 4030612631 ;

Fax: +91403548271.

E-mail: subhadra@lvpei.org

Received: 23 February 2015 Accepted in revised form: 4 September 2015

Published online:

23 October 2015

\begin{abstract}
Purpose To characterise and differentiate posterior microphthalmos (PM) and nanophthalmos (NO) using morphometric parameters.

Patients and methods Consecutive case database of patients with hyperopia $>+7.00 \mathrm{D}$ sphere was analysed retrospectively for clinical and biometric characterisation. Thirtyeight consecutive high-hyperopic subjects (75 eyes) with axial lengths $<20.5 \mathrm{~mm}$ underwent uniform comprehensive ocular evaluation. Twenty-five subjects were diagnosed as PM and 13 as NO based on the horizontal corneal diameter. Parameters analysed included visual acuity, refraction, horizontal corneal diameter, anterior chamber depth, lens thickness, axial length, fundus changes, and associated ocular pathology. Primary outcome measures: ocular biometry difference between PM and NO. Secondary outcome measures: differences in associated ocular pathologies between PM and NO.

Results Hyperopia ranged from +7 to $+17 \mathrm{D}$ and was similar in the two groups. Lens thickness was statistically more in NO than in PM group $(4.53 \pm 0.75 \mathrm{~mm} v \mathrm{~s}$ $3.82 \pm 0.48 \mathrm{~mm}, P<0.001)$, whereas anterior chamber depth was more in the PM than in NO group $(3.26 \pm 0.36 \mathrm{~mm}$, vs $2.59 \pm 0.37 \mathrm{~mm}$, $P<0.001)$. NO had higher association with angle-closure glaucoma ( $66.7 \%$ vs $0 \%$ ) and pigmentary retinopathy ( 38.5 vs $\mathbf{8 . 0 \%}$ ) but lesser association with macular folds ( $0 \%$ vs $24 \%$ ) as compared with PM. NO was associated with poorer visual acuity.
\end{abstract}

N Relhan', S Jalali', N Pehre², HL Rao ${ }^{3}$,

U Manusani ${ }^{1}$ and L Bodduluri ${ }^{1}$
Conclusion PM and NO have significant differences in lens thickness, anterior chamber depth, prevalence of glaucoma, pigmentary retinopathy, macular pathology, and visual acuity while being similar in hyperopic refraction.

Eye (2016) 30, 120-126; doi:10.1038/eye.2015.206; published online 23 October 2015

\section{Introduction}

Microphthalmos is a developmental ocular disorder characterised by an eye with a total axial length at least $2 \mathrm{SD}$ below the mean for that age group. ${ }^{1}$ Earliest mention and classification of microphthalmos have been by Duke Elder in 1964 where it has been classified into simple/ pure and complex/complicated types. ${ }^{2}$ Simple or pure type are those with no malformations and the complex type have ocular associations including colobomas, cysts, tunica vasculosa lentis, corectopia, aniridia, persistent pupillary membrane, and so on. ${ }^{3-5}$ These changes occur owing to faulty development of the optic vesicle.

Clinical variants of microphthalmos range from gross reduction in overall size of the globe (nanophthalmos; NO) or shortening of either the anterior segment (relative anterior microphthalmos) or posterior segment (posterior microphthalmos; (PM) of eyeball.

$\mathrm{NO}$ is an anomaly described as having microphthalmos, ${ }^{6}$ smaller corneal diameter, and a high hyperopia of $>+8.00 \mathrm{D}$ sphere. ${ }^{7}$ Various other ocular findings reported include microcornea, a high lens/eye volume ratio, 
angle-closure glaucoma (ACG), ${ }^{8}$ yellow macular pigmentation, chorioretinal folds, macular hypoplasia, retinal cysts, ${ }^{9}$ crowded optic discs, ${ }^{9}$ retinal striae and folds, ${ }^{10}$ wrinkling of ILM, ${ }^{11}$ thickened sclera with abnormal collagen fibrils, ${ }^{12}$ recurrent or persistent choroidal effusions, ${ }^{2,6}$ and non-rhegmatogenous retinal detachments. ${ }^{11}$

Eyes with short axial length but normal corneal diameter were first reported in $1975^{13}$ and the term 'PM' was introduced to differentiate these patients from $\mathrm{NO}$ that have small corneal diameter. Clinical features reported in PM include reduced axial length with a normal-sized cornea and normal anterior segment and a disproportionately small size of the posterior segment. Other characteristics described include high hyperopia, uveal effusion, elevated papillomacular retinal fold, ${ }^{14,15}$ fine retinal striae, tapetoretinal degenerations similar to fundus flavimaculatus and fundus albipunctatus, retinoschisis, absence or marked reduction of the foveal capillary-free zone, pseudopapilledema, and macular holes. ${ }^{15,16}$

Cases of high hyperopia may in some instances be cases of PM, which go unrecognised because of normal-sized corneas. Thus biometric measurements are essential for the diagnosis of this entity. It has been mostly reported to coexist with several other ophthalmic conditions and to occur as non-familial sporadic cases. PM is a more recently described clinical entity as compared with NO. Owing to overlapping clinical features and different time line descriptions of these two hyperopic entities, the literature is not clear regarding detailed characterisation and differentiation of these two conditions and their associations. Prospectively collected database of hyperopes with well-defined definitions and detailed ocular evaluation would help in better characterisation of $\mathrm{PM}$ and NO. We report our findings of such a database collected from the year 2000 to 2010.

\section{Materials and methods}

From the year 2000 we maintained a database of all cases of high hyperopia (defined as hyperopia $>+7.00 \mathrm{D}$ ) seen in our vitreoretinal service at L V Prasad Eye Institute.

The institute review board gave consent for the database analysis and reporting. After signed consent by patient or legal guardians in case of minors, all subjects underwent detailed comprehensive eye examination. The database was reviewed and analysed for the following parameters for the current report: visual acuity, recorded by Snellen visual acuity chart converted to Log MAR value, Cycloplegic refraction, using Haag-Streit streak retinoscope, and A-scan Biometry using Lenstar LS900. The adjustable length of the light beam of the Haag-Streit slit lamp was used to measure the horizontal Corneal diameter from 'white to white' of the cornea. ${ }^{1}$ Fundus photographs taken on the Zeiss FP 450 plus fundus camera after pupillary dilatation were reviewed and analysed. History of nyctalopia, consanguinity, familial nature of the condition, any associated ocular pathology, or any systemic disease was analysed. The associated pathologies detected were treated according to preferred practice and accepted guidelines in the institute, including in-house low vision and rehabilitation management and are not reported here. All measurements were done by certified Optometry faculty in the department. All analysis and interpretation of the findings were done by the Ophthalmologists (NR, SJ, NP, HLR).

Inclusion criteria: high hyperopia $>+7.00 \mathrm{D}$ sphere with axial length $<20.5 \mathrm{~mm}$.

Exclusion criteria: any patient who did not provide written consent for detailed examination or where records were incomplete.

Our primary outcome measures were the ocular biometry difference between PM and NO. The secondary outcome measures included any differences in associated ocular pathologies between PM and NO.

\section{Statistical analysis}

Descriptive statistics included mean and SD for normally distributed variables and median, first quartile, and third quartile values for non-normally distributed variables. All continuous variables have been represented using mean and SD. Shapiro-Wilk test was used to test the distribution of the variables. Wilcoxon signed rank test was used to test the differences between continuous variables and $\chi^{2}$-test for categorical variables between the $\mathrm{PM}$ and NO groups. Statistical analyses were performed using commercial software (Stata ver. 11.0; StataCorp, College Station, TX, USA). A $P$-value of $\leq 0.05$ was considered statistically significant.

\section{Results}

Between the years 2000 to 2010, the data of 38 highhyperopic subjects having axial length $\leq 20.5 \mathrm{~mm}$ with complete clinical details was available for the study. Patients were defined to have PM if the horizontal corneal diameter was $\geq 11 \mathrm{~mm}$ and NO if the horizontal corneal diameter was $<11 \mathrm{~mm}$. For age $<1$ year, an axial length of $<19.2 \mathrm{~mm}$ was considered. ${ }^{2}$

Based on these criteria, PM was present in 25 subjects and NO in 13 subjects. Table 1 gives details of various parameters in the two groups. 
Table 1 Comparative analysis of the two groups

\begin{tabular}{|c|c|c|c|}
\hline & $\begin{array}{l}\text { Posterior microphthalmos (25 patients, } \\
49 \text { eyes) }\end{array}$ & Nanophthalmos (13 patients, 26 eyes) & P-value \\
\hline Males: females & $12(48 \%): 13(52 \%)$ & $8(61.54 \%): 5(38.46 \%)$ & \\
\hline Age at presentation & $16.02 \pm 15.34$ years & $33.20 \pm 19.31$ years & 0.006 \\
\hline $\begin{array}{l}\text { Mean best-corrected log MAR } \\
\text { visual acuity (Snellen's) }\end{array}$ & $\begin{array}{c}0.61 \pm 0.38 \log \text { MAR }(20 / 80) \\
\text { range } 0-1.3 \log \text { MAR } \\
(20 / 20-20 / 400)\end{array}$ & $\begin{array}{c}0.95 \pm 0.74 \log \text { MAR }(20 / 200) \\
\text { range } 0.3-3 \log \text { MAR } \\
(20 / 40-\mathrm{HM})\end{array}$ & 0.004 \\
\hline Corneal diameter (mm) & $\begin{array}{c}11.39 \pm 0.38 \\
\text { (range } 11-12 \text { ) }\end{array}$ & $\begin{array}{c}10.07 \pm 0.18 \\
\text { (range } 10-10.5 \text { ) }\end{array}$ & $<0.001$ \\
\hline Anterior chamber depth (mm) & $\begin{array}{c}3.20 \pm 0.39 \\
\text { (range } 2.43-4.03 \text { ) }\end{array}$ & $\begin{array}{c}2.68 \pm 0.42 \\
\text { (range 1.97-3.34) }\end{array}$ & $<0.001$ \\
\hline Lens thickness (mm) & $\begin{array}{l}3.93 \pm 0.48 \\
\text { (range 3.1-4.94) }\end{array}$ & $\begin{array}{c}4.77 \pm 0.55 \\
\text { (range } 4.66-5.48 \text { ) }\end{array}$ & $<0.001$ \\
\hline Axial length (mm) & $\begin{array}{c}17.91 \pm 1.82 \\
\text { (range } 15.12-20.5 \text { ) }\end{array}$ & $\begin{array}{c}17.20 \pm 1.64 \\
\text { (range } 15.38-20.49)\end{array}$ & 0.05 \\
\hline Keratometry (dioptres) & $\begin{array}{l}\text { Mean } 46.01 \mathrm{D}^{\mathrm{a}} \\
(41.75-53.5)\end{array}$ & $\begin{array}{l}\text { Mean } 48.00 \mathrm{D}^{\mathrm{b}} \\
(44.18 \mathrm{D}-51.9 \mathrm{D})\end{array}$ & 0.18 \\
\hline Refractive error (dioptres) & $\begin{array}{l}11.59 \pm 3.28 \\
(+7 \text { to }+17 \mathrm{D})\end{array}$ & $\begin{array}{c}11.30 \pm 3.94 \\
(+7 \text { to }+17 \mathrm{D})\end{array}$ & 0.93 \\
\hline Consanguinity & $12(48 \%)$ & $7(53.85 \%)$ & 0.833 \\
\hline Familial & $10(40 \%)$ & 7 (53.85\%) & 0.50 \\
\hline Nyctalopia & $4(16 \%)$ & $4(30.77 \%)$ & 0.32 \\
\hline Angle-closure glaucoma & $0^{c}$ & $\begin{array}{l}9(69.23 \%) \\
\text { (6 angle-closure glaucoma, } 3 \text { narrow angles with } \\
\text { iridotomy done) }\end{array}$ & $<0.001$ \\
\hline Congenital anomaly & $\begin{array}{l}\text { Atrial septal defect with patent } \\
\text { ductus arteriosus- } 1\end{array}$ & None & \\
\hline Macular folds & $24 \%$ (6 patients, 12 eyes) & $0 \%$ & $<0.001$ \\
\hline Pigmentary retinopathy & $8 \%$ (2 patients) & $38.6 \%$ (5 patients) & $<0.001$ \\
\hline
\end{tabular}

${ }^{a} 48$ eyes. ${ }^{b} 23$ eyes. 'One eye had open-angle glaucoma for which trabeculectomy was done.

\section{Demography}

Forty-nine eyes of 25 patients (12 males and 13 females) with PM (one eye was not included as axial length was $22.96 \mathrm{~mm}$ ) and 26 eyes of 13 patients ( 8 males and 5 females) with NO were analysed. Mean age at presentation was lower in PM $(16.02 \pm 15.34$ years, range 6 months to 72 years) than in NO group $(33.20 \pm 19.31$ years, range 3-60 years). Age distribution at presentation is shown in Figure 1. Eighty percent of PM presented at $<20$ years of age, whereas only $23 \%$ of NO presented in the first two decades (Figure 1).

\section{Best-corrected visual acuity}

(Tables 1 and 2) Out of 49 eyes the visual acuity data were available for 47 PM eyes (excluding two eyes of a 6-month baby). Mean visual acuity in PM group was $0.61 \pm 0.38 \log$ MAR (Snellen's equivalent - 20/80) and ranged from 0 to 1.3 log MAR (Snellen's equivalent: 20/20-20/400).

Among 26 eyes with NO, 4 had visual acuity of perception of light only and one was PL negative. The mean visual acuity in the remaining 21 eyes was $0.95 \pm 0.74 \log$ MAR (Snellen's equivalent - 20/200) and ranged from 0.3 to $3 \log$ MAR (Snellen's equivalent 20/40 to Hand motions). The Table 2 shows the comparative visual acuity in the two groups.

\section{Refraction}

(Tables 1 and 3) Mean refractive error (cycloplegic objective retinoscopy in Dioptres) of 48 eyes (one pseudophakic eye data not available) with PM was $+11.59 \pm 3.28 \mathrm{D}$ (range +7 to $+17 \mathrm{D}$ ), whereas in 23 eyes (one phthisis and two with no retinoscopic reflex excluded) with $\mathrm{NO}$, refraction was+11.30 $\pm 3.94 \mathrm{D}$ (range +7 to $+17 \mathrm{D}$ ). The refractive error in both groups was comparable. Table 3 shows the distribution of refractive errors in the two groups.

\section{Biometric parameters}

(Table 1) The mean axial length of the 49 eyes with PM was $17.91 \pm 1.82 \mathrm{~mm}$ (range 15.12-20.50 mm), whereas in 25 eyes with $\mathrm{NO}$ (excluding one eye with phthisis bulbi) it was $17.20 \pm 1.64 \mathrm{~mm}$ (range $15.38 \pm 20.49 \mathrm{~mm}$ ). The infant of age $<6$ months had axial length of $19.2 \mathrm{~mm}$ in the two 


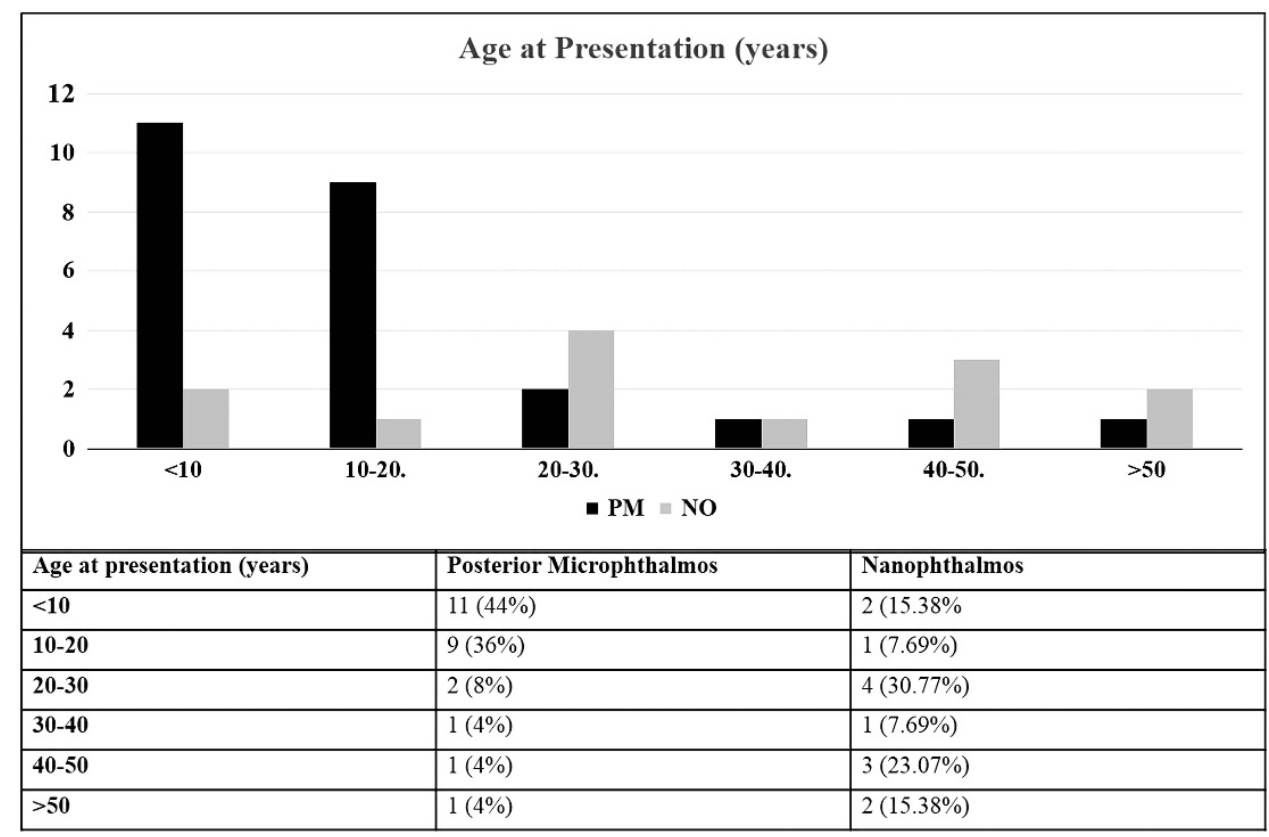

Figure 1 Distribution of posterior microphthalmos (PM) and nanophthalmos (NO) according to age at presentation. PM group presented at a relatively lower age.

Table 2 Comparative visual acuity data of posterior microphthalmos and nanophthalmos

\begin{tabular}{lcc}
\hline Visual acuity & $\begin{array}{c}\text { Posterior } \\
\text { microphthalmos N (\%) } \\
47 \text { eyes }^{\mathrm{a}}\end{array}$ & $\begin{array}{c}\text { Nanophthalmos N (\%) } \\
26 \text { eyes }\end{array}$ \\
\hline $20 / 20-20 / 40$ & $13(27.66 \%)$ & $2(7.69 \%)$ \\
$>20 / 40-20 / 100$ & $20(42.55 \%)$ & $11(42.31 \%)$ \\
$>20 / 100-\mathrm{PL}$ & $14(29.79 \%)$ & $13(50 \%)$
\end{tabular}

aTwo eyes of a baby excluded as Snellen visual acuity could not be measured.

eyes. The mean corneal diameter in PM group was $11.39 \pm 0.38 \mathrm{~mm}$ (range 11-12 mm), whereas in NO group was $10.07 \pm 0.18 \mathrm{~mm}$ (range $10-10.5 \mathrm{~mm}$ ). The range of keratometry in PM group was 41.75-53.5 D (mean $46.01 \mathrm{D})$, whereas in NO group was 44.18 D-51.19 D (mean $48 \mathrm{D}$ ). The anterior chamber depth in PM ranged from 2.43 to $4.03 \mathrm{~mm}$ (mean $3.20 \mathrm{~mm}$ ), whereas in NO it was $1.97-3.34 \mathrm{~mm}$ (mean $2.68 \mathrm{~mm}$ ). The range of lens thickness in PM was 3.1-4.94 (mean $3.93 \mathrm{~mm}$ ), whereas in NO the range was $4.66-5.48$ (mean $4.77 \mathrm{~mm}$ ).

Associated findings. In the PM group, one eye had openangle glaucoma for which trabeculectomy was done. Ten patients had familial disease, whereas 12 patients came from parental consanguineous marriages. Four patients had history of nyctalopia and only two patients had pigmentary retinopathy clinically.
Table 3 Magnitude of refractive error in posterior microphthalmos and nanophthalmos

\begin{tabular}{lcr}
\hline $\begin{array}{l}\text { Refractive error } \\
\text { range }\end{array}$ & $\begin{array}{c}\text { Posterior } \\
\text { microphthalmos N (\%) } \\
48 \text { eyes }\end{array}$ & $\begin{array}{c}\text { Nanophthalmos N (\%) } \\
23 \text { eyes }\end{array}$ \\
\hline$<10 \mathrm{D}$ & $23(47.92 \%)$ & $14(63.87 \%)$ \\
$10-15 \mathrm{D}$ & $16(33.33 \%)$ & $4(17.39 \%)$ \\
$>15 \mathrm{D}$ & $9(18.75 \%)$ & $5(21.74 \%)$ \\
\hline
\end{tabular}

Among 13 NO patients, 9 patients had ACG or narrow angles (six ACG, three narrow angles for which iridotomy was done). Seven patients had familial disease and parental consanguinity was present in seven patients. Four patients had nyctalopia, whereas pigmentary retinopathy was present in five patients. Macular abnormality (presence of folds at macula) was seen in $24 \%$ of PM vs none in NO group. Figure 2 shows representative fundus photographs of PM and NO. The retinal structural and functional aspects of these entities were evaluated by OCT and ERG and will be reported in part II.

Comparative data analysis (Tables $1-3$, and Figure 1) revealed that the PM and NO groups differed in terms of mean age at presentation, best-corrected visual acuity, anterior chamber depth, lens thickness, keratometry, and association with glaucoma or macular folds but not in the magnitude of the refractive error. Better visual acuity was seen in PM group with 70\% of PM eyes compared with $50 \%$ of NO eyes having Snellen's visual acuity better than 20/100 (Table 2). 

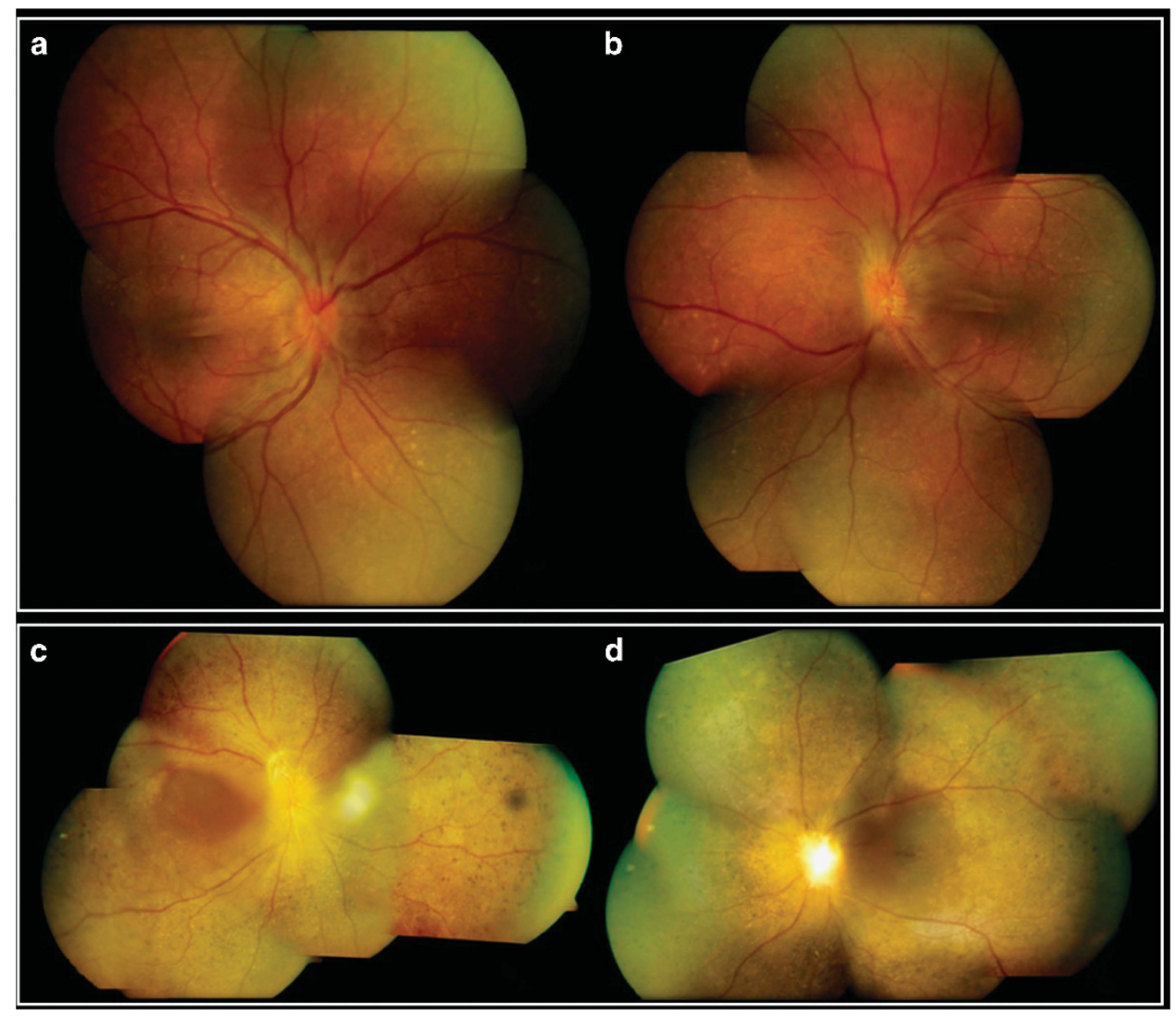

Figure 2 Fundus photograph of right eye (a) and left eye (b) of patient with posterior microphthalmos show the presence of macular fold whereas fundus photograph of right eye (c) and left eye (d) of a patient with nanophthalmos show characteristic features of retinitis pigmentosa (bony spicule pigmentation, disc pallor and arteriolar attenuation).

\section{Discussion}

This is the largest series of consecutive patients from a prospective database of high hyperopia analysed with the objective of characterising the two distinct clinical entities of PM and NO primarily on biometric measurements. Various studies published at different time points have used different and sometimes overlapping biometric criteria to define these two clinical entities. ${ }^{6-16}$ We used a single measurement of horizontal corneal diameter of $<11 \mathrm{~mm}$ for differentiating NO from PM in simple microphthalmic eyes with axial length of $<20.5 \mathrm{~mm}$ in our high-hyperopia cohort.

Detailed biometry evaluation of the two groups revealed many interesting findings. The magnitude of refractive error in the two groups was similar (Table 3) and hence cannot be used to differentiate these two entities. However, significant differences were seen in other biometric measures including thicker lenses and shallow AC depth in NO, whereas eyes with PM had normal anterior chamber depths and lens thickness (normal anterior chamber depth 3.14-3.6 mm, ${ }^{17,18}$ whereas normal lens thickness ranges from $4-4.45 \mathrm{~mm}^{19}$ ).
As nanophthalmic eyes had small globes with thicker lenses, their anterior chambers were also small (Table 1), predisposing them to the angle closure as was evident from our finding of around 70\% ACG/angle closure in our patients with NO. Such angle closure/ACG was not seen in any of our 49 eyes with PM, though one eye did undergo treatment for open-angle glaucoma. This difference is clinically of great significance in identifying hyperopic eyes at risk of ACG because preventive iridotomy could be considered in such eyes to prevent vision loss.

Age at presentation in our study was lower in PM compared with NO group. The difference in the age at presentation has not been described in literature. It seems that though both PM and NO groups have high hyperopia and poor uncorrected vision of early onset, patients with PM tended to present to our tertiary-care center at an early age. This could be either because the referring ophthalmologist/optometrist noted the abnormal macular folds or because the visual acuity could not be improved with glasses in these young patients with macular pathology. On the other hand the NO group seems to have had good visual acuity in younger age with glasses and lost visual acuity later in life owing to 
glaucoma or the progressive pigmentary retinopathies and hence presented to our center only later in life. This is reflected in the much poor presenting visual acuity in the NO group then the PM group in our series.

In our cohort of patients, $\sim 24 \%$ patients of PM group had macular structural abnormalities as compared with none in the NO group. The NO group had predominance of patients with progressively vision-threatening abnormalities like pigmentary retinopathies in $38.46 \%$ $(5 / 13)$ patients compared with only $8 \%(2 / 25)$ patients in the PM group. The association of pigmentary retinopathy with microphthalmos (both NO and PM) has been well documented in case reports and case series. ${ }^{20,21}$ Cases of syndromes of pigmentary retinopathy, optic disk drusen, and foveoschisis with both entities of $\mathrm{PM}$ and $\mathrm{NO}^{7,22}$ are also reported but were not seen in our current series of cases. Familial association of pigmentary retinopathy has been reported rarely. ${ }^{15}$ In our study, the pigmentary retinopathy was familial in two of the $13 \mathrm{NO}$ patients $(15.38 \%)$. A number of our patients in the current series had familial PM (40\%) or NO (53.85\%) and there was high prevalence of consanguinity in the parents though these two factors were not inter-related. Our group has earlier reported a family with pigmentary retinopathy and PM syndrome ${ }^{23}$ that is not included in the current study.

Nowilaty et al $^{17}$ recently reported an inverse correlation between axial length and corneal power in patients with PM and noted steeper corneas in PM eyes. Our cohort of patients also showed steep corneas (mean keratometry >46 D; normal value 43-44 D) in both PM and NO groups. However, we could not detect any significant correlation between axial length and corneal power in our patients $\left(r^{2}\right.$ was 0.03 and 0.10 for PM and NO group, respectively, $P=0.66)$. Nowilaty et $a l^{17}$ also found a positive correlation between axial length and corneal diameter, which could not be confirmed in our data $\left(r^{2}\right.$ was 0.12 and 0.02 for PM and NO group, respectively, $P=0.66)$.

In conclusion, PM patients presented early in life with high hyperopia and better visual acuities as compared with patients with NO. Eyes with PM had normal anterior chamber depths and lens thickness with low association of angle closure. The PM eyes were often associated with structural retinal abnormalities such as macular folds and rarely with pigmentary retinopathy. $\mathrm{NO}$ patients had shallow anterior chamber depth and more lens thickness with higher association with progressive conditions such as glaucoma and pigmentary retinopathy. Both groups had steep corneas. A clinician who encounters hyperopia $>+7.00 \mathrm{D}$ needs to record the axial length and the horizontal corneal diameter to diagnose and differentiate these two clinical entities. They need to be aware that PM and NO can be differentiated by detailed biometry and associated pathologies can be detected by detailed comprehensive eye examination. When dealing with patients having these abnormal syndromes, the current data can provide information to patients and relatives regarding the relative probability of familial condition, glaucoma, and vision-threatening pigmentary retinopathy.

\section{Summary}

What was known before

- Scant data available regarding morphometric features of PM and NO.

- Considerable overlap in literature as the two terminologies were described temporally apart.

What this study adds

- High-hyperopia database analysis morphometric characterisation of PM and NO.

- Risk factors and how to differentiate and evaluate and follow-up such entities.

\section{Conflict of interest}

The authors declare no conflict of interest.

\section{Acknowledgements}

No external funding support was obtained for the purpose of this study. The study was supported by Hyderabad eye research foundation. There are no financial disclosures to be made. Contribution of authors -design and conduct of the study (SJ); collection (SJ, UM, LB), management (SJ), analysis and interpretation of the data (SJ, NR, HLR); manuscript preparation (SJ, NR, UM, LB, NP), review (SJ, NR, HLR, NP), or approval of the manuscript (SJ, NR, HLR, NP,UM,LB). IRB approval was obtained before the analysis of the data.

\section{References}

1 Elder MJ. Aetiology of severe visual impairment and blindness in microphthalmos. Br J Ophthalmol 1994; 78(5): 332-334.

2 Singh OS, Sofinski SJ. Anomalies in the size of the eyeIn. In: Duke-Elder S (ed). System Of Ophthalmology. CV Mosby: St Louis, USA, 1963, pp 488-495.

3 Weiss AH, Kousseff BG, Ross EA, Longbottom J. Simple microphthalmos. Arch Ophthalmol 1989; 107(11): 1625-1630.

4 Vingolo EM, Steindl K, Forte R, Zompatori L, Iannaccone A, Sciarra A et al. Autosomal dominant simple microphthalmos. J Med Genet 1994; 31(9): 721-725.

5 Weiss AH, Kousseff BG, Ross EA, Longbottom J. Complex microphthalmos. Arch Ophthalmol 1989; 107(11): 1619-1624.

6 Ryan EA, Zwaan J, Chylack LT. Nanophthalmos with uveal effusion: clinical and embryologic considerations. Ophthalmology 1982; 89(9): 1013-1017. 
7 Crespí J, Buil JA, Bassaganyas F, Vela-Segarra JI, DíazCascajosa J, Ayala-Ramírez R et al. A novel mutation confirms MFRP as the gene causing the syndrome of nanophthalmos-renititis pigmentosa-foveoschisis-optic disk drusen. Am J Ophthalmol 2008; 146(2): 323-328.

8 Mandal AK, Das T, Gothwal VK. Angle closure glaucoma in nanophthalmos and pigmentary retinal dystrophy: a rare syndrome. Indian J Ophthalmol 2001; 49(4): 271-272.

9 MacKay CJ, Shek MS, Carr RE, Yanuzzi LA, Gouras P. Retinal degeneration with nanophthalmos, cystic macular degeneration, and angle closure glaucoma. A new recessive syndrome. Arch Ophthalmol 1987; 105(3): 366-371.

10 Serrano JC, Hodgkins PR, Taylor DS, Gole GA, Kriss A. The nanophthalmic macula. Br J Ophthalmol 1998; 82(3): 276-279.

11 Buys YM, Pavlin CJ. Retinitis pigmentosa, nanophthalmos, and optic disc drusen: a case report. Ophthalmology 1999; 106(3): 619-622.

12 Yue BY, Kurosawa A, Duvall J, Goldberg MF, Tso MO, Sugar J. Nanophthalmic sclera. Fibronectin studies. Ophthalmology 1988; 95(1): 56-60.

13 Boynton JR, Purnell EW. Bilateral microphthalmos without microcornea associated with unusual papillomacular retinal folds and high hyperopia. Am J Ophthalmol 1975; 79(5): 820-826.

14 Ryckewaert M, Zanlonghi X, Bertrand-Cuignet $\mathrm{H}$, Constantinides G. High hyperopia with papillomacular fold. Ophthalmologica 1992; 204(1): 49-53.

15 Spitznas M, Gerke E, Bateman JB. Hereditary posterior microphthalmos with papillomacular fold and high hyperopia. Arch Ophthalmol 1983; 101(3): 413-417.
16 Lee S, Ai E, Lowe M, Wang T. Bilateral macular holes in sporadic posterior microphthalmos. Retina 1990; 10(3): 185-188.

17 Nowilaty SR, Khan AO, Aldahmesh MA, Tabbara KF, Al-Amri A, Alkuraya FS. Biometric and molecular characterization of clinically diagnosed posterior microphthalmos. Am J Ophthalmol 2013; 155(2): 361-372.e7.

18 Nguyen ATQ, Johnson MA, Hutcheson KA. Good visual function in posterior microphthalmos. J AAPOS 2000; 4(4): 240-242.

19 Adler FH. Physiology of the eye: Clinical Application. 4th edn. Mosby-Year Book: St Louis, MO, USA, 1965.

20 Khairallah M, Messaoud R, Zaouali S, Ben Yahia S, Ladjimi A, Jenzri S. Posterior segment changes associated with posterior microphthalmos. Ophthalmology 2002; 109(3): 569-574.

21 Proença H, Castanheira-Dinis A, Monteiro-Grillo M. Bilateral nanophthalmos and pigmentary retinal dystrophyan unusual syndrome. Graefes Arch Clin Exp Ophthalmol 2006; 244(9): 1203-1205.

22 Ayala-Ramirez R, Graue-Wiechers F, Robredo V, Amato-Almanza M, Horta-Diez I, Zenteno JC. A new autosomal recessive syndrome consisting of posterior microphthalmos, retinitis pigmentosa, foveoschisis, and optic disc drusen is caused by a MFRP gene mutation. Mol Vis 2006; 12: 1483-1489.

23 Pehere N, Jalali S, Deshmukh H, Kannabiran C. Posterior microphthalmos pigmentary retinopathy syndrome. Doc Ophthalmol 2011; 122(2): 127-132. 